\title{
Genetic Analysis of Congenital Cystic Adenomatoid Malformation Reveals a Novel Pulmonary Gene: Fatty Acid Binding Protein-7 (Brain Type)
}

\author{
AMY J. WAGNER, AMBER STUMBAUGH, ZACHARY TIGUE, JESS EDMONDSON, AGNES C. PAQUET, DIANA L. FARMER, \\ AND SAMUEL HAWGOOD
}

\begin{abstract}
Departments of Surgery [A.J.W., D.L.F.], Pediatrics [A.S., Z.T., J.E., S.H.], and Medicine [A.C.P.], University of California, San Francisco, San Francisco, California 94143; Department of General Surgery [A.J.W.], Virginia Mason Medical Center, Seattle, Washington 98101
\end{abstract}

\begin{abstract}
The pathogenesis of congenital cystic adenomatoid malformation (CCAM) is unknown and its natural history is unpredictable. Fatty acid binding protein-7 (FABP-7) has been previously described in brain and breast development, but never before in the lung. We investigate gene expression in CCAM, and hypothesize that CCAM results from an aberration in the signaling pathway during lung development. Under IRB approval, tissue specimens of fetal CCAM, fetal control, postnatal CCAM, and postnatal control were examined and microarray analysis was performed. Candidate differentially expressed genes were selected with log-odds ratio (B) $>0$ and false discovery rate $<0.05$. Validation of differential expression was achieved at the RNA and protein levels. FABP-7 was underexpressed in fetal CCAM compared with fetal lung in both the microarray and by RT-PCR. Findings were duplicated by Western Blot analysis and immunohistochemistry. This is the first description of FABP-7 in the human lung. Decreased expression of FABP-7 in fetal CCAM compared with normal fetal lung at both the RNA and protein levels suggests FABP-7 may have a role in pulmonary development and in the pathogenesis of CCAM. (Pediatr Res 64: 11-16, 2008)
\end{abstract}

$\mathrm{C}$ ongenital cystic adenomatoid malformation (CCAM) is a multicystic pulmonary mass characterized by an abnormal proliferation of tissue resembling terminal respiratory bronchioles. The relatively rare lesions usually occur unilaterally and involve only one lobe of the lung. Diagnosis is often made by prenatal ultrasonography.

CCAM has a variable natural history. Some lesions regress in utero with no residual abnormality at birth (1-2), others are associated with fetal polyhydramnios, mediastinal shift, and nonimmune hydrops fetalis resulting in the risk of fetal demise (3). Mediastinal shift and hydrops fetalis are indications for fetal intervention.

Received November 28, 2007; accepted February 26, 2008.

Correspondence: Amy J. Wagner, M.D., Department of Graduate Medical Education, Virginia Mason Medical Center, H8-GME, 925 Seneca Street, Seattle, WA 98101; e-mail: amy.wagner@vmmc.org

This study was supported by in part by the UCSF NHLBI Shared Microarray Facility (NIH grant HL072301) and by the General Clinical Research Center at San Francisco General Hospital (RR-00083).
Stocker et al.'s original classification of CCAM included three subtypes based predominantly on the cyst size. Type I lesions are composed of single or multiple large cysts $(>2 \mathrm{~cm}$ in diameter); type II lesions are multiple, smaller cysts $(<1 \mathrm{~cm}$ in diameter); and type III are more solid, microcystic lesions. Two variants were later added to the original classification system: type 0 , composed of bronchial-like histology and type IV, a more distal acinar lesion (4).

The pathogenesis of CCAM is unclear. Its original description was that of a hamartomatous lesion. The five Stocker CCAM types have also been described as each being distinct malformations, with unique etiologies resulting from a localized obstruction or atresia (2). The parenchymal maldevelopment characterizing CCAM has been associated with bronchial atresia (5). The bronchial atresia may result from a localized arrest in the bronchial tree during the branching phase of lung development, the pseudoglandular phase.

Lung development during the pseudoglandular phase involves extensive epithelial-mesenchymal interaction of growth factors, transcription factors, and signaling molecules. Previous studies evaluating the pathogenesis of CCAM, have focused on one individual developmental factor $(6-10)$. These studies have further been limited by confounding variables that occur as CCAM tissues acquired from infancy or childhood were compared with control lung samples not of the same age, but to fetal lung tissue or lung tissue from other species. Lung development is a complex process, in which one single signaling molecule has many downstream effects, thus examination of a single factor is not sufficient. Therefore, our investigation began with examination of the complete gene expression profile with microarray analysis.

\section{MATERIALS AND METHODS}

Human subjects. Under University of California San Francisco (UCSF) IRB approval, fetal CCAM samples $(n=4)$, postnatal CCAM samples $(n=$

Abbreviations: CCAM, congenital cystic adenomatoid malformation; FABP-7, fatty acid binding protein-7; CC10, clara cell 10 kilodalton protein 
5), and postnatal control samples $(n=3)$ were acquired at the time of surgical resection. Fetal CCAM samples were resected because of the findings of hydrops. Fetal control samples $(n=2)$ were obtained from mid-gestation fetuses immediately after laminaria-primed dilation and evacuation was performed. All samples were taken from the distal parenchyma away from the hilum. Samples were flash frozen in liquid nitrogen and stored at $-80^{\circ} \mathrm{C}$ for further analysis. The Cooperative Human Tissue Network (funded by the National Cancer Institute) provided an additional control sample $(n=1)$. Fetal and postnatal CCAM samples were verified by the UCSF Department of Pathology.

Tissue histology. Tissue for immunohistochemistry was fixed in a solution of $4 \%$ paraformaldehyde in $0.1 \mathrm{M}$ phosphate buffer, $\mathrm{pH} 7.4$, submerged in a $30 \%$ sucrose solution overnight at $4^{\circ} \mathrm{C}$, and OCT (Tissue Tek; Miles, Elkhart, IN) frozen in liquid nitrogen. Cryosections were cut $2 \mu \mathrm{m}$ thick and applied to Superfrost Plus slides (Fisher Scientific; Fair Lawn, NJ). Tissue was immersed in $0.3 \%$ Triton solution in $0.1 \%$ bovine serum albumin or phosphate-buffered saline, $10 \%$ goat serum in phosphate-buffered saline or phosphate-buffered saline, and primary antibody in goat serum solution overnight at $4{ }^{\circ} \mathrm{C}$. The following antibodies were used: rabbit polyclonal fatty acid binding protein-7 (FABP-7) (1:1000, gift from Rosaline Godbout, The Cross Cancer Institute, University of Alberta, Canada); monoclonal mouse $\alpha$-smooth muscle actin (1:50; Dako; Carpinteria, CA); monoclonal mouse PE-CAM-1 (1:20; Dako; Carpinteria, CA); monoclonal mouse CD-68 (1:100; Dako, Clone KP1; Carpinteria, CA); goat polyclonal CC10 (1:200; Santa Cruz Biotechnology, Inc, Santa Cruz, CA); HT $_{\text {I } 56}$, a mouse MAb directed against an integral membrane protein specific in human lung to type I

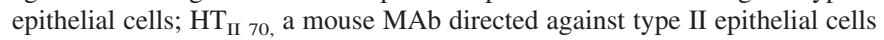
(Dobbs, unpublished data). Proteins were visualized by incubating sections with goat anti-rabbit or goat anti-mouse IgG conjugated to Alexa 594 or Alexa 488 at 1:3000 (Molecular Probes Inc., Carlsbad, CA) and mounted with ProLong antifade agent (Molecular Probes Inc). Oil Red O staining (American Mastertech Scientific, Lodi, CA) was completed at $60^{\circ} \mathrm{C}$ for 6 min after slides were immersed in propylene glycol for $2 \mathrm{~min}$. Fluorescence and phase contrast images were captured with a Leica DC500 camera on a Leica Orthoplan microscope.

RNA extraction and preparation for Affymetrix microarray. One hundred to $150 \mathrm{mg}$ of tissue were homogenized and total RNA extracted using Qiagen RNeasy Midiprep protocol (Qiagen, Valencia, CA). Total RNA was quantified by spectrophotometry at 260 or $280 \mathrm{~nm}$ and the quality assessed by Agilent Bioanalyzer (Agilent Technologies, Palo Alto, CA). For further analysis, samples were required to meet the standards of the UCSF Genomics Core Group and have appropriate 18S and 28S ribosomal RNA chromographs. Acceptable samples were amplified, Biotin-labeled, and fragmented. The quality was reassessed by the Agilent Bioanalyzer and only samples meeting quality standards were hybridized. HG-U133 Plus 2.0 Affymetrix microarray chips were hybridized with $10 \mu \mathrm{g}$ cRNA and processed according to the manufacture's protocol (Affymetrix, Foster City, CA). Hybridization signals were detected in the BioConductor.

Microarray data analysis. Images were analyzed using Affymetrix GeneChip Operating System software. Assessment of hybridization quality was performed using BioConductor package affyPLM $(11,12)$. Preprocessing of all 14 arrays was performed using Robust Multichip Average algorithm (13). Differential expression was assessed using a linear model. For each probe set, fold-change and odds ratio (probability of being differentially expressed or probability of not being differentially expressed) were calculated using functions of the BioConductor package limma. Genes with $\log 2$ odds ratio (B-statistic) greater than 0 were considered as statistically significant. Gene Ontology analysis was performed using Ingenuity Pathways Analysis database (Ingenuity Systems, Redwood City, CA.).

Lung cRNA was obtained from subjects in all four groups using a standardized approach and analyzed on microarrays containing 54,675 probe sets. Hierarchical clustering was used to group samples based on the pattern of expression of the 200 most variable genes. The groups were first examined to determine whether each sample within the group were similar. This was completed by a pair wise comparison of log intensity for samples from each CCAM group compared with the appropriate control group for all probe sets. Fetal CCAM had more similarity to its controls than the postnatal CCAM had to its controls. The pair wise comparison for the fetal CCAM to fetal control group yielded a correlation of $0.97-0.99$ for each comparison; the pair wise comparison for the postnatal CCAM to the postnatal control group yielded a correlation of $0.94-0.99$.

Genes were ranked using moderated t-statistic (limma) and were selected with log-odds ratio (B) $>0$ as candidate differentially expressed genes in each group vs. the appropriate control. We examined other significance cutoffs by controlling for the false discovery rate at $5 \%$ level (Table 1). As no gene was statistically significant in the comparison of the postnatal CCAM vs. controls, the differentially expressed genes list was generated by selecting genes with
Table 1. Microarray results: differentially expressed genes

\begin{tabular}{lccr}
\hline & Abs & FDR & \\
& $(\mathrm{M})>1$ & $<0.05$ & B $>0$ \\
\hline Fetal CCAM vs. fetal control (no. genes) & 849 & 2 & 43 \\
Postnatal CCAM vs. postnatal control & 774 & 0 & 0 \\
Postnatal CCAM $v$ s. fetal CCAM & 1470 & 600 & 608 \\
\hline
\end{tabular}

absolute log-ratios greater than 2 (more than 4-fold difference). Therefore, the focus was placed on the fetal CCAM vs. fetal control group.

The candidate differentially expressed genes in the fetal CCAM vs. fetal control groups were selected based on B $>0$, and ordered by $\mathrm{M}$ values. Genes were also plotted based on $\mathrm{M}$ values (log ratios between the fetal CCAM and fetal control) and median log intensity (A values).

Statistical analysis. Assessment of hybridization was performed using BioConductor quality control (affyPLM algorithm). Hierarchical clustering was performed using Pearson correlation. Confounding variables were examined for using hierarchical clustering of genes selected based on variance. All 14 arrays were preprocessed using Robust Multichip Average algorithm from BioConductor. Genes were ranked using moderated t-statistic (limma). Candidate differentially expressed genes were selected with log-odds ratio (B) $>0$ and false discovery rate at 0.05 . Gene Ontology analysis was performed using Ingenuity Pathways Analysis database (Ingenuity Systems, Redwood City, CA.).

Real time quantitative PCR. RNA was extracted using Qiagen RNeasy Midiprep (Qiagen, Valencia, CA). Total RNA (1 $\mu \mathrm{g} / \mathrm{sample})$ was reverse transcribed using RETROscript reagents (Ambion, Austin, TX), substituting random hexamers for random decamers. Real-time quantitative PCR amplification of total cDNA was performed using an ABI PRISM 7900HT Sequence Detector System with a 384 well block (Applied Biosystems, Foster City, CA). TaqMan Gene Expression Assay primers and TaqMan $2 \times$ Universal PCR Master Mix (Applied Biosystems, Foster City, CA) were used. Standard curves were created from serial dilutions of a cDNA pool of several experimental samples and all samples were amplified in triplicate and quantified using the relative standard curve method. Control reactions were performed for each sample without reverse transcriptase.

Sequencing of amplification product. FABP-7 primers were designed in regions distinct from the FABP-3 or FABP-5 sequence. The forward primer was AAAGAGGGGAAAGGGCAAGG; the reverse primer was CCGACCAGGAACATTTTTATGC for a product size of 435 base pairs. PCR amplification of total cDNA was performed for $95^{\circ} \mathrm{C}$ for $3 \mathrm{~min}, 54^{\circ} \mathrm{C}$ for $20 \mathrm{~s}$, $72^{\circ} \mathrm{C}$ for $50 \mathrm{~s}, 95^{\circ} \mathrm{C}$ for $20 \mathrm{~s}$, repeated for a total of 30 times, and $72^{\circ} \mathrm{C}$ for 10 min. The product was sequenced by the Biomolecular Resource Center at UCSF.

Western blot analysis. For each sample, $100 \mathrm{mg}$ of tissue was sonicated in $1 \mathrm{~mL}$ of buffer, centrifuged, then $20 \mu \mathrm{g}$ of protein was subjected to sodium dodecyl sulfate-polyacrylamide gel electrophoresis using $15 \%$ Tris- $\mathrm{HCl}$ gel (Bio-Rad Laboratories, Hercules, CA). The protein was transferred to a nitrocellulose membrane (PROTRAN, Schleicher and Schuell Bioscience), blocked with 5\% powdered milk, and then incubated with FABP-7 antibody (1:500); goat anti-rabbit IgG-HRP (1:3000, Chemicon International, Temecula, CA). Bands were developed using ECL Plus Western Blotting Detection System (GE Healthcare, Buckinghamshire, UK) and chemiluminescence was detected using Kodak XAR film.

\section{RESULTS}

Sample characteristics. Gene expression of CCAM was examined in fetal samples and from pediatric specimens. Fetal CCAM samples were compared with age and gender-matched fetal lung controls, and postnatal CCAM samples were compared with normal postnatal control lung. Fourteen microarrays were completed. Samples were divided into four groups: Fetal CCAM samples $(n=4)$, fetal control samples $(n=2)$, postnatal CCAM samples $(n=5)$, and postnatal control samples $(n=3)$ (Table 2).

Microarray analysis. The candidate differentially expressed genes in the fetal CCAM $v s$. fetal control groups were selected based on $\mathrm{B}>0$, ordered by $\mathrm{M}$ values, and plotted based on $\mathrm{M}$ 
Table 2. Sample characteristics

\begin{tabular}{llll}
\hline \multicolumn{1}{c}{ Fetal CCAM } & Fetal control & Postnatal CCAM & $\begin{array}{c}\text { Postnatal } \\
\text { control }\end{array}$ \\
\hline 20-wk male (type I) & 20-wk male & 2-d male (type II) & 1-d male \\
26-wk female (type I) & 24-wk female & 3-wk male (type I) & 1-y male \\
27-wk female (type II) & & 2-mo male (type I) & 7-y male \\
28-wk female (type III) & & 1-y male (type II) & \\
& & 1.5 -y male (type I) & \\
& &
\end{tabular}

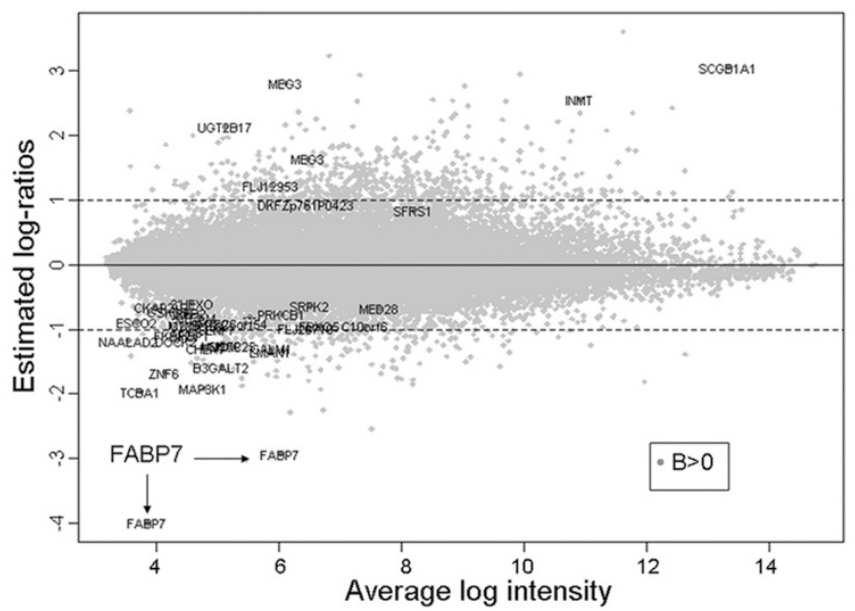

Figure 1. Scatter plot between estimated $M$ values (log ratios between genes expressed in fetal CCAM and genes expressed in fetal controls) and A values (median log intensity).

values (log ratios between the fetal CCAM and fetal control) and A values (median log intensity) (Fig. 1).

Of the differentially expressed genes, the genes with the most distinct differential expression were CC10 (SCGB1A1 secretoglobin, family $1 \mathrm{~A}$, member 1 ; CCSP or CC10), and FABP-7, (brain). CC10 (Clara cell $10 \mathrm{kD}$ protein) is a secretory protein with specificity to Clara cells. CC10 was found to have an $\mathrm{M}$ value of 3.055 (which is an 8.31-fold difference in expression) in the fetal CCAM compared with the fetal control lung tissue (Figs. 1 and 2).

FABP-7 is a member of a family of cytoplasmic proteins that bind long-chain fatty acids and has a role in fatty acid uptake, transport, and metabolism. The expression of FABP-7 had an $\mathrm{M}$ value of -3.994 in fetal CCAM compared with fetal control lung, meaning CCAM lung had a 16-fold decreased expression of FABP-7 compared with normal control lung. Other genes involved in pulmonary development were examined, but did not meet statistical significance (Figs. 1 and 2).

RNA validation. CDNA microarray revealed that FABP-7 is underexpressed in fetal CCAM compared with fetal lung by a 16-fold difference. Quantitative RT-PCR of FABP-7 expression validated these findings. Fetal FABP-7 was also underexpressed in fetal CCAM lung compared with fetal control lung by a 12 -fold difference (Fig. 2). In the array, CC10 was over-expressed in the fetal CCAM compared with control lung by a difference of 8 -fold. The quantitative PCR data confirmed these findings as well, as CC10 was overexpressed by a 139-fold difference in fetal CCAM compared with control lung.

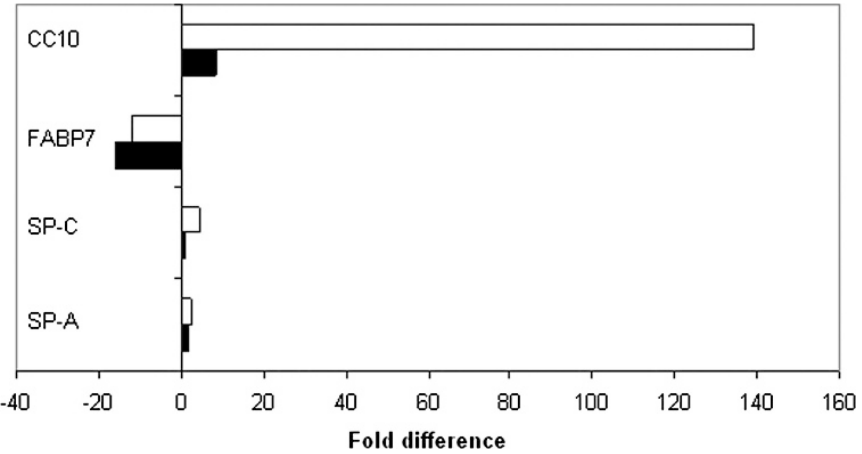

Figure 2. Gene expression of CCAM vs. control. Quantitative RT-PCR validation of microarray analysis results $(\square)$ and PCR results $(\square)$ for each gene.

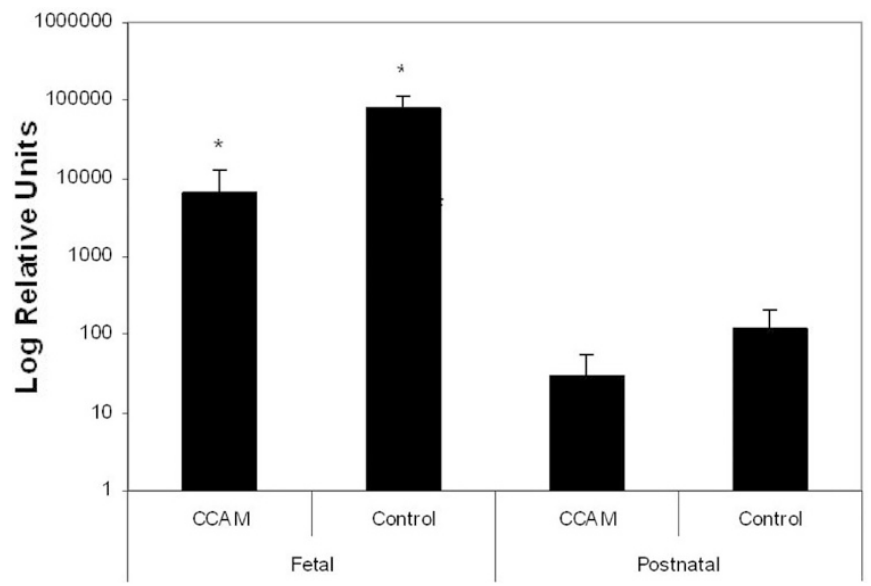

Figure 3. Gene expression of FABP-7 by RT-PCR in fetal CCAM vs. fetal control, and in postnatal CCAM vs. postnatal control. Data presented as means \pm SEM, and displayed logarithmically. $\left({ }^{*} p=0.036\right)$.

Additionally, postnatal CCAM and control lung tissue were examined for differential expression of FABP-7 and CC10. Expression of $\mathrm{CC} 10$ paralleled the fetal data, with the expression increased in the postnatal CCAM compared with the postnatal control lung by a 39 -fold difference (Fig. 2). There was also a decrease in expression of FABP-7 by postnatal CCAM lung compared with the control lung by a 4-fold difference (Fig. 3).

To confirm that the amplified PCR product being analyzed was indeed FABP-7, we designed primers unique for FABP-7 with the sequence being distinct from FABP-3 or FABP-5. The analyzed sequence had 100\% homology to FABP-7 and no significant similarity to FABP-3 or FABP-5.

Protein validation. Western blot analysis was performed and validated findings of FABP-7 expression in the fetal lung. FABP-7 protein is expressed in fetal lung, and at higher levels than in fetal CCAM (Fig. 4).

Immunohistochemistry was completed to further examine the expression of FABP-7 in the fetal lung. FABP-7 appeared to be expressed by a mesenchymal cell of the fetal lung at 20 and $24 \mathrm{wk}$ gestation, and at higher levels in control tissue than in CCAM. There was no expression visualized at 4 mo of age, or in adult lung tissue. The cell expressing FABP-7 appears in the alveolar septa and is 1.5-2 times the size of an erythrocyte. The cells exhibit nuclear staining with indistinct cytoplasmic 


\section{CCAM}

$\begin{array}{llllll}\text { A1 } & \text { A2 } & \text { A3 } & \text { A4 } & \text { C1 } & \text { C2 }\end{array}$

\section{$14.3 \mathrm{kD}$}

Figure 4. Expression of FABP-7 protein by Western Blot analysis. Lane A1, fetal CCAM, 27 wk gestation; Lane A2, fetal CCAM, 28 wks; Lane A3, fetal CCAM, 26 wks; Lane A4, fetal CCAM, 20 wks; Lane C1, fetal control lung, 20 wks; Lane $C 2$, fetal control lung, 24 wks. Bands visualized at $14.3 \mathrm{kD}$.
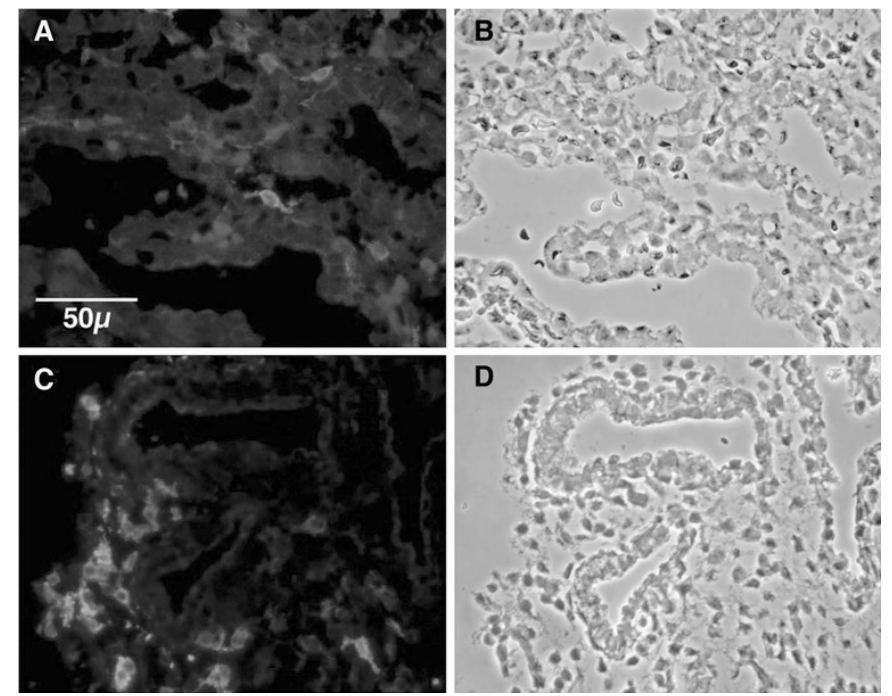

Figure 5. (A) Immunohistochemistry of 20-wk CCAM fetal lung stained for FABP-7; $(B)$ phase view; $(C)$ 20-wk control fetal lung stained for FABP-7; (D) phase view $(40 \times)$.
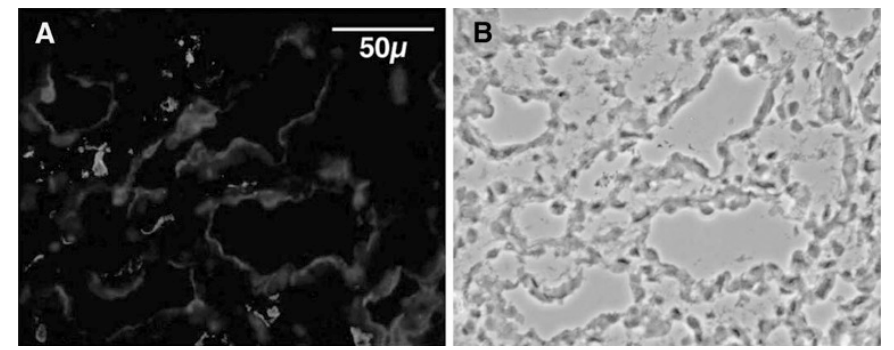

Figure 6. (A) Immunohistochemistry of 24-wk control fetal lung double stained for FABP-7 and Type I epithelial cells $\left(\mathrm{HT}_{\mathrm{I}}\right)(40 \times) ;(B)$ Phase view $(40 \times)$.

borders. The staining is moderate in intensity. The intensity was similar in both groups, but there were more cells that expressed FABP-7 in the fetal control tissue than the fetal CCAM tissue (Fig. 5).

The cell expressing FABP-7 appears to be in the mesenchyme (Fig. 6), but did not appear to colocalize with other mesenchymal cell markers. We stained cells for PE-CAM (an endothelial cell marker), CD-68 (a macrophage marker), $\alpha$-smooth muscle actin, and Oil Red-O (both markers of fibroblasts). The epithelium was identified by staining for type I and type II pneumocytes with $\mathrm{HT}_{\mathrm{I}}$ and $\mathrm{HT}_{\mathrm{II}}$.

\section{DISCUSSION}

CCAM appears to be an aberration in normal lung development. In our microarray analysis, we found two candidate genes that met the most stringent criteria for differential expression: CC10 and FABP-7. CC10 expression has previously found to be increased in CCAM (7), and our study confirmed this. FABP-7 has not been previously implicated in the pathogenesis of CCAM or in pulmonary development, and therefore was the focus of our study.

The pseudoglandular, or branching, phase of pulmonary development occurs between 6 and $16 \mathrm{wk}$ of gestation. It involves a myriad of signaling molecules regulated spatially and temporally, which allow extensive communication between pulmonary epithelium and mesenchyme. Some signaling factors involved in the bidirectional epithelialmesenchymal interactions include members of the Fibroblast growth factor (FGF), Hedgehog, Wingless, transforming growth factor- $\beta$, and bone morphogenic protein signaling pathways (14-17). One or more of these factors could be involved in the pathogenesis of CCAM, as the disease occurs during the pseudoglandular phase of lung development.

Previous studies have focused on a single factor during this elaborate epithelial-mesenchymal interaction. For example, platelet-derived growth factor BB, expression was found to be increased in fetal CCAM samples compared with control fetal lung (8). Platelet-derived growth factor BB is a mesenchymal growth factor, which increases cell proliferation potentially through downstream effectors such as FGF-7 or transforming growth factor- $\beta$. However, there was no difference found in expression of the downstream factor FGF-7 (KGF).

Hox genes code for transcription factors that regulate lung development during branching morphogenesis. Hoxb-5 protein expression was examined in postnatally resected CCAM compared with human and mouse fetal lung controls. Levels of Hoxb-5 protein in CCAM tissue were maintained at levels similar to the pseudoglandular and canalicular stage of lung development, again supporting the hypothesis of arrested development (9).

Glial cell-derived neurotrophic factor (GDNF) expression in postnatally resected CCAM, normal fetal lung, and normal postnatal lung was examined. GDNF was expressed in both epithelial and endothelial cells of normal fetal lung, but no expression was found in normal postnatal lung (10). GDNF stimulates proliferation, and its temporal expression pattern may imply a role in lung development.

These published studies of gene expression in CCAM support the hypothesis that the pathogenesis is due to an aberration in lung development. However, they examined one individual signaling molecule in the extensive network of epithelial-mesenchymal interactions. Our study is the first of gene expression profiling using microarray analysis to examine the differential expression of genes in fetal CCAM compared with appropriate fetal controls.

The microarray results revealed what appears to be a novel pulmonary gene expressed during development. FABP-7 belongs to a family of intracellular lipid-binding proteins with molecular masses of $14-15 \mathrm{kD}$. A variety of functions have been attributed to these proteins, including the transport of long-chain fatty acids, targeting of fatty acids to specific metabolic pathways, and the subsequent regulation of gene expression and cell growth (18). 
FABP-7 is also called Brain-FABP, as it was first described in the murine brain. The human FABP-7 gene has been cloned and mapped to chromosome 6q22-23 (19). It is involved in the spatial development of the cortex, and is strongly expressed in radial glial cells. Here it has a strong affinity for docosahexaenoic acid, a long-chain fatty acid that is an important nutrient for neural tissues. Addition of anti FABP-7 antibodies to mixed cultures of glia and cerebellar neurons inhibited neuronal migration, suggesting FABP-7 influences the correct migration of developing neurons into cortical layers (18).

FABP-7 is also expressed in breast tissue, where it is known as mammary-derived growth-inhibitor related gene. In the breast, FABP-7 has a role in development and differentiation of lactiferous ducts. Overexpression of FABP-7 in transgenic mice resulted in increased ductal differentiation (20). The lungs were not examined.

Although other members of the FABP family have been described previously in the lung, FABP-7 has not. Both FABP-3 (heart type, or H-FABP) and FABP-5 (epidermal type, or E-FABP) were found by RT-PCR to be expressed in the lung $(21,22)$. Northern blot analysis showed H-FABP to be restricted to lung fibroblasts, and E-FABP to be present in type II cells, lung fibroblasts, and in macrophages (22). FABP-7 expression was examined at weeks 9-10 of human fetal lung, and found not to be expressed (19).

FABP-3 and FABP-5 are known to be expressed in the human fetal lung and have a role in pulmonary development, specifically in surfactant synthesis. Fatty acids are required for the biosynthesis of phospholipids, particularly dipalmitoyl phosphatidylcholine, required in the production of surfactant by type II pneumocytes in the lung. FABP may transport the precursor fatty acid for phospholipid synthesis. A FABP-5/ FABP-3 double-knockout mouse was generated, and type II cells were analyzed from the wildtype and double-knockout mice. The double-knockout mice resulted in impaired surfactant synthesis with a decrease in palmitate uptake, $\beta$-oxidation, and incorporation of neutral lipids and phosphatidylcholine of type II cells (22).

Phosphatidylcholine synthesis is dependent on cholinephosphate cytidylyltransferase, the rate-limiting enzyme. Fatty acids have been shown to activate cholinephosphate cytidylyltransferase (23). FABPs in the cytoplasm bind free long-chain fatty acids. FABP-7 has a greater propensity to bind longchain unsaturated fatty acids over saturated fatty acids (24) and may have a role in surfactant synthesis.

FABP-7 may also have a role in pulmonary development by an effect on the glucocorticoid pathway. Fatty acids have been shown to modulate glucocorticoid receptor binding. In L2 cells, a rat type II cell line, long-chain unsaturated fatty acids markedly inhibited steroid binding to the glucocorticoid receptor. Long-chain saturated fatty acids had no effect (25). Further analysis on L2 cells and kinetics analysis on brain tissue revealed that unsaturated fatty acids modulated the receptor by a noncompetitive type of inhibition; the receptor was modulated by binding to sites different from the glucocorticoid binding sites (26).

Glucocorticoids have multiple effects on development, including the acceleration of lung maturation and stimulation of surfactant synthesis (27). After the glucocorticoid ligand binds to the receptor, the receptor translocates from the cytoplasm to the nucleus, where it serves either directly or indirectly as a transcriptional regulator. FABP-7 modulates the concentration of free fatty acid (FFA) in the cytoplasm. With a high concentration of FFA in the cytoplasm, the glucocorticoid receptor undergoes a confirmational change making it unresponsive to glucocorticoids. This could have an impact on lung development.

There is an observed relationship between corticosteroid administration and regression of CCAM lesions. Patients with CCAM and nonimmune hydrops fetalis with predicted mortality did not undergo fetal intervention, and were treated instead with prenatal betamethasone to increase lung maturity. After a course of prenatal steroids during the second trimester, patients had resolution of hydrops and were delivered without respiratory distress in most cases $(28,29)$. The exact mechanism of the improvement in these patients' pulmonary status and resolution of CCAM after steroid administration is unknown.

We have shown that fetal CCAM expresses significantly less FABP-7 than control fetal lung. Less FABP-7 may result in less long-chain unsaturated fatty acids being bound to the protein, with more free in the cytoplasm. Unsaturated long chain fatty acids are known to cause inhibition of the glucocorticoid receptor. Therefore, CCAMs, with low FABP-7 and high FFA, would have less glucocorticoid response than normal lung. Without the glucocorticoid effect on the lung, a resulting arrest in development could occur.

There are some potential limitations to our findings. First, both CCAM and normal pulmonary tissue consist of many diverse tissue types. We were unable to quantify the relative proportions of each different tissue type sampled in each group or account for possible differences in the components of each group, which could contribute to differences in gene expression. Additionally, another difference between the control and CCAM groups is the presence of hydrops. We were unable to obtain control tissue in hydropic fetuses as only diseased lung was surgically removed. This may also be a confounding variable, which could contribute to differences in gene expression.

FABP-7 is a novel pulmonary protein whose expression has previously never been described in the lung. There is decreased expression of FABP-7 in fetal CCAM compared with normal fetal lung at both the RNA and protein levels. FABP-7 may have a role in pulmonary development, in the pathogenesis of CCAM, and is the focus of further research.

Acknowledgments. The authors thank Dr. Rosaline Godbout for generously providing us with the FABP-7 antibody.

\section{REFERENCES}

1. MacGillivray TE, Harrison MR, Goldstein RB, Adzick NS 1993 Disappearing fetal lung lesions. J Pediatr Surg 28:1321-1324

2. Miller JA, Corteville JE, Langer JC 1996 Congenital cystic adenomatoid malformation in the fetus: natural history and predictors of outcome. J Pediatr Surg 31:805-808

3. De Santis M, Masini L, Noia G, Cavaliere AF, Oliva N, Caruso A 2000 Congenital cystic adenomatoid malformation of the lung: antenatal ultrasound findings and fetal-neonatal outcome. Fifteen years of experience. Fetal Diagn Ther 15:246-250 
4. Stocker JT, Madewell JE, Drake RM 1977 Congenital cystic adenomatoid malformation of the lung. Classification and morphologic spectrum. Hum Pathol 8:155-171

5. Riedlinger WF, Vargas SO, Jennings RW, Estroff JA, Barnewolt CE, Lillehei CW, Wilson JM, Colin AA, Reid LM, Kozakewich HP 2006 Bronchial atresia is common to extralobar sequestration, intralobar sequestration, congenital cystic adenomatoid malformation, and lobar emphysema. Pediatr Dev Pathol 9:361-373

6. Cangiarella J, Greco MA, Askin F, Perlman E, Goswami S, Jagirdar J 1995 Congenital cystic adenomatoid malformation of the lung: insights into the pathogenesis utilizing quantitative analysis of vascular marker CD34 (QBEND-10) and cell proliferation marker MIB-1. Mod Pathol 8:913-918

7. Morotti RA, Cangiarella J, Gutierrez MC, Jagirdar J, Askin F, Singh G, Profitt SA, Wert SE, Whitsett JA, Greco MA 1999 Congenital cystic adenomatoid malformation of the lung (CCAM): evaluation of the cellular components. Hum Pathol 30:618625

8. Liechty KW, Crombleholme TM, Quinn TM, Cass DL, Flake AW, Adzick NS 1999 Elevated platelet-derived growth factor-B in congenital cystic adenomatoid malformations requiring fetal resection. J Pediatr Surg 34:805-809

9. Volpe MV, Pham L, Lessin M, Ralston SF, Bhan E, Cutz E, Nielsen HC 2003 Expression of Hoxb-5 during human lung development and in congenital lung malformations. Birth Defects Res A Clin Mol Teratol 67:550-556

10. Fromont-Hankard G, Philippe-Chomette P, Delezoide AL, Nessmann C, Aigrain Y, Peuchmaur M 2002 Glial cell-derived neurotrophic factor expression in normal human lung and congenital cystic adenomatoid malformation. Arch Pathol Lab Med $126: 432-436$

11. Bolstad BM, Collin F, Simpson KM, Irizarry RA, Speed TP 2004 Experimental design and low-level analysis of microarray data. Int Rev Neurobiol 60:25-58

12. Gentleman RC, Carey VJ, Bates DM, Bolstad B, Dettling M, Dudoit S, Ellis B, Gautier L, Ge Y, Gentry J, Hornik K, Hothorn T, Huber W, Iacus S, Irizarry R, Leisch F, Li C, Maechler M, Rossini AJ, Sawitzki G, Smith C, Smyth G, Tierney L, Yang JY, Zhang J 2004 Bioconductor: open software development for computational biology and bioinformatics. Genome Biol 5:R80

13. Irizarry RA, Bolstad BM, Collin F, Cope LM, Hobbs B, Speed TP 2003 Summaries of affymetrix genechip probe level data. Nucleic Acids Res 31:e15

14. Hogan BL, Yingling JM 1998 Epithelial/mesenchymal interactions and branching morphogenesis of the lung. Curr Opin Genet Dev 8:481-486

15. Mendelson CR 2000 Role of transcription factors in fetal lung development and surfactant protein gene expression. Annu Rev Physiol 62:875-915
16. Chuang PT, McMahon AP 2003 Branching morphogenesis of the lung: new molecular insights into an old problem. Trends Cell Biol 13:86-91

17. Cardoso WV, Lu J 2006 Regulation of early lung morphogenesis: questions, facts and controversies. Development 133:1611-1624

18. Haunerland NH, Spener F 2004 Fatty acid-binding proteins-insights from genetic manipulations. Prog Lipid Res 43:328-349

19. Godbout R, Bisgrove DA, Shkolny D, Day RS 1998 Correlation of B-FABP and GFAP expression in malignant glioma. Oncogene 16:1955-1962

20. Wang M, Lui YE, Goldberg ID, Shi YE 2003 Induction of mammary gland differentiation in transgenic mice by the fatty acid-binding protein MRG. J Biol Chem 278:47319-47325

21. Paulussen RJ, Geelen MJ, Beynen AC, Veerkamp JH 1989 Immunochemical quantitation of fatty-acid-binding proteins. Tissue and intracellular distribution, postnatal development and influence of physiological conditions on rat heart and liver FABP. Biochim Biophys Acta 1001:201-209

22. Guthmann F, Hohoff C, Fechner H, Humbert P, Borchers T, Spener F, Rustow B 1998 Expression of fatty-acid-binding proteins in cells involved in lung-specific lipid metabolism. Eur J Biochem 253:430-436

23. Burkhardt R, Von Wichert P, Batenburg JJ, Van Golde LM 1988 Fatty acids stimulate phosphatidylcholine synthesis and CTP: choline-phosphate cytidylyltransferase in type II pneumocytes isolated from adult rat lung. Biochem J 254:495-500

24. Balendiran GK, Schnutgen F, Scapin G, Borchers T, Xhong N, Lim K, Godbout R, Spener F, Sacchettini JC 2000 Crystal structure and thermodynamic analysis of human brain fatty acid-binding protein. J Biol Chem 275:27045-27054

25. Viscardi RM, Max SR 1993 Unsaturated fatty acid modulation of glucocorticoid receptor binding in L2 cells. Steroids 58:357-361

26. Kato J, Takano A, Mitsuhashi N, Koike N, Yoshida K, Hirata S 1987 Modulation of brain progestin and glucocorticoid receptors by unsaturated fatty acid and phospholipid. J Steroid Biochem 27:641-648

27. Ballard PL, Ballard RA 1986 Hormones and Lung maturation, Monographs on Endocrinology. Springer-Verlag: New York, pp 197-236

28. Tsao K, Hawgood S, Vu L, Hirose S, Sydorak R, Albanese CT, Farmer DL, Harrison MR, Lee H 2003 Resolution of hydrops fetalis in congenital cystic adenomatoid malformation after prenatal steroid therapy. J Pediatr Surg 38:508-510

29. Peranteau WH, Wilson RD, Liechty KW, Johnson MP, Bebbington MW, Hedrick HL, Flake AW, Adzick NS 2007 Effect of maternal betamethasone administration on prenatal congenital cystic adenomatoid malformation growth and fetal survival. Fetal Diagn Ther 22:365-371 